\title{
Astronomy, technology development and industry
}

\author{
Laurent Vigroux ${ }^{1,2}$ \\ ${ }^{1} \mathrm{DSM} / \mathrm{IRFU} / \mathrm{CEA}$, \\ CE Saclay, 91191 Gif-sur-Yvette Cedex, France \\ ${ }^{2}$ Institut d'Astrophysique de Paris, \\ 98 bis, Boulevard Arago, 75014 Paris, France \\ email: vigroux@iap.fr
}

\begin{abstract}
Astronomy is perhaps the best example of fundamental research aiming to increase our knowledge well beyond our human neighborhood. But astronomy is also a Big Science, which is partly technology-driven. Progress in observational capabilities is due to progress in detectors, telescopes, satellites, etc. I use three examples -radio astronomy, adaptive optics and detectorsto describe the complex interactions between astronomy, technology development and industry. I conclude by a short description of the global economic impact of astronomy.
\end{abstract}

Keywords. technology transfer, adaptive optics, detectors, radio astronomy, research and development

\section{Introduction}

Fundamental research is generally associated with pure science, aiming to increase our knowledge of Nature, without aims at applications. A distortion of this definition would be an intellectual game played by selfish individuals, disconnected from real life. Unfortunately many people, including some politicians, share this hostile vision. And when Big Science, like particle physics or astronomy, are scrutinised, the same persons explain that fundamental research deals with very expensive toys and that public money should be spent on more fruitful activities. On the opposite, the impact of fundamental research on the society as a whole, including its economical importance, is recognised by many governments, which use fundamental research as a development tool to improve their economy. Astronomy is a good case to understand the complicated relationship between science, technology and industry.

Dealing with the Universe as a whole, its contents and its governing laws, astronomy is a perfect example of pure science. But astronomy is also strongly dependant on technological developments. In the last 50 years, large telescopes, radio interferometers, satellites, powerful computers and networks have been at the origin of a revolution in our understanding of astronomical objects. In that sense, astronomy is technology-driven. The return from astronomy to technology developments and industry is less well known, and often ignored. In this review, I describe the complex interaction between astronomy and technology developments in industry with three examples: radio astronomy, adaptive optics and detectors. I conclude by a short description of the global economic impact of astronomy. 


\section{The early days of radio astronomy}

The first detection of a radio emission from an astronomical object was fortuitous. In 1930, Bell Telephone Laboratories wanted to develop wireless transatlantic communications. One engineer, Karl Jansky, was asked to study parasitic emission in the frequency range around $20 \mathrm{MHz}$, a high frequency not well known at that time. For this purpose, Jansky built a mobile antenna supported by front-wheels and axles from an old Ford $\mathrm{T}$ and started looking for emission sources in the sky. He immediately discovered the emission by storms, but he was surprised by a faint periodic signal, with a period approximately one day. Later, he was able to demonstrate that the periodicity was only 23 hours and 56 minutes, which is exactly the period of the apparent rotation of a star in the sky. This demonstrated that it was a source of celestial origin. He eventually found that the source was associated with the Milky Way and also that the signal increased when moving towards the Galactic center. Despite his own interests to search for other astronomical sources, he was assigned new tasks by Bell Labs and could not continue to develop radio astronomy. This discovery gained a large attention from the astronomers and the public, but no astronomer was interested to continue in this new domain $\dagger$.

Grote Reber was a radio-engineer who wanted to go beyond the first discovery of Jansky. He failed to find somebody supporting this activity. He took a full-time job in a radio company in Chicago, and decided to build with his own funds a radio telescope in the backyard of his house. It was the first parabolic reflector antenna. Working at night and during holidays, he was able to confirm the discovery of Jansky in 1938 and, later on, to make the first map of the Milky Way in radio waves. The origin of the Milky Way radio emission remained a mystery for several years, until it was demonstrated in the 60s that it is due to synchrotron emission of cosmic ray electrons moving in the Galactic magnetic field. While synchrotron emission was observed in an accelerator in 1947, the theory of synchrotron emission has benefited from all the work done to understand the radio emission of celestial sources, in particular in the Soviet Union, with the works of Ginzburg and co-workers. Synchrotron beams are now common tools to study the properties of materials, especially in molecular biology.

The great Dutch astronomer Jan Oort was very interested by the findings of Reber. He was working on the structure of the Galaxy. His work was hampered by interstellar dust causing visible light extinction, preventing a uniform access to every region of the Galaxy, and distorting the distance scale. Oort realised that the interstellar matter is transparent to radio waves, and that by using a line emission that could be detected in the radio wave domain he could perform velocity measurements using the Doppler effect everywhere in the Galaxy. He asked one of his students, H.C. van de Hulst, to identify suitable lines and to verify that they could be detected. Van de Hulst calculated that the best line should be an atomic Hydrogen transition at $21 \mathrm{~cm}$. Using a 7.5-m diameter radar previously used by the Germans on the Atlantic coastline during the war, the Dutch group started observations to detect the $21 \mathrm{~cm}$ line. Due to the lack of sensitivity of their detectors, they were unable to detect it. At the same time two physicists in the US, Harold Ewen and Edward Purcell, aware of van de Hulst's work, started building a system with a horn and detector specifically optimised for the detection of the $21 \mathrm{~cm}$ line. Purcell had a good experience of radio techniques, having worked on radars during the war. He was to win a Nobel Prize for his work on nuclear magnetic resonance. To improve the sensitivity of the detectors, they used a new modulation scheme by switching frequencies. Thanks to this new technique, they detected the $21 \mathrm{~cm}$ line for the first time. Then the Dutch group adopted the frequency switching method and confirmed the Ewen

$\dagger$ see www.nrao.edu/index.php/learn/radioastronomy/radioastronomyhistory 
and Purcell detection. The two results were published in the same issue of Nature in 1951. This result was confirmed almost simultaneously by an Australian group (see, e.g. van Woerden \& Strom 2006).

These three stories are good examples of the interaction of technology development and astronomy. In the three cases, the discovery was possible only thanks to a technical progress. But the discovery of Jansky was serendipitous, Reber's map of the Milky Way was obtained to get better observations, but without a supporting theory, while the 21 $\mathrm{cm}$ line detection was due to a theoretical prediction and the design of an instrument dedicated to this specific observation.

Since its beginnings, radio astronomy has had very strong links with technical progress, and it is still the case (Lequeux 2005). Radio telescopes and their associated instrumentation are challenging technically and equipments for radio astronomy have been at the forefront of performances in many areas: high-gain antennas in mm- and cm- domains, low-noise and high-gain amplifiers, digital processing and massive data analysis. It should also be noted that radio astronomers have invented and were the first to make extensive use of aperture synthesis imaging techniques to make wide-field maps at radio frequencies. All the software developed for radio interferometers were at the base of the systems developed later for X-ray medical imaging. The aperture synthesis technique is also central for medical tomography or brain imaging.

\section{Adaptive optics: crossing roads for astronomical and defense applications}

Looking for the first time through the eyepiece of a large telescope for is always disappointing. The images seem blurred and have a fast erratic motion. This is due to atmospheric turbulence, which distorts the images. In principle, a 4-m telescope limited only by optical diffraction would provide an angular resolution of $0{ }^{\prime \prime} 1$. Atmospheric blurring, a phenomenon called seeing by astronomers, degrades the angular resolution at around $1^{\prime \prime}$ in good sites and under good meteorological conditions. This problem was well known for a long time and a concept to compensate for the astronomical seeing was even proposed by Horace Babcock in 1953 (Babcock 1953). Unfortunately, this concept was unachievable with the technology known at that time, and no real attempts were made until the $70 \mathrm{~s} \dagger$.

In the meantime the opening of space following the successful launch of Sputnik in 1957 triggered a strong interest for spy satellites. Between 1960 and 1972, the USA launched 146 spy satellites. And it was probably the same in the Soviet Union, even though we still do not know the exact number of spy satellites launched. It became very important to watch carefully the satellites in orbit. Ground-based telescopes looking on a satellite orbiting well above the atmosphere face the same problem of image degradation as when looking at more distant stars. The seeing problem became common to astronomy and defense. Defense-oriented research was also engaged to find focusing techniques for laser beams on distant objects, which require also to compensate for the laser beam widening through the atmosphere. With their limited budget, astronomers developed techniques with a posteriori image processing, while defense engineers tackled the problem of active correction of the telescope optics to ensure real-time compensation of the atmospheric image degradation. They developed new systems based on wavefront sensors and deformable mirrors. The first imaging system providing a $2 \mathrm{D}$ adaptive correction was set up on an

$\dagger$ see e.g. Claire Max (2001) cfao.ucolick.org/EO/resources/History_AO_Max.pdf 
Air Force telescope in Hawaii in 1982. In 1983, Ronald Reagan launched the Strategic Defense Initiative, and all information on adaptive optics became strictly classified.

During the next 10 years, the development of adaptive optics remained modest in astronomy. A crucial step was achieved at the beginning of the 90s thanks to the declassification of some adaptive optics components, and the availability of a new generation of infrared detectors. Since then, adaptive optics systems have been developed actively, with improved performances and users-friendly interfaces. Adaptive optics is now an essential part of modern telescopes to the extent that the future generation of Extremely Large Telescopes, with diameters of $30 \mathrm{~m}$ or larger, cannot be operated without an efficient adaptive optics systems. The needs for astronomy and defense have now diverged. Astronomy has more interests in partial correction in the infrared domain and on a somewhat large field to observe faint stars and distant galaxies, while defense applications require high-adaptive performances in the visible and with a very rapid response time. Defense experts are also still working on the development of airborne laser systems to destroy ballistic missiles or ground targets.

Adaptive optics has now important applications in opthalmology. The inhomogeneity in the eye lens and in the cornea causes blurred images on the retina. The image degradation process is similar to the atmospheric seeing degradation. It can be corrected with adaptive optics systems very similar to the cameras in use on telescopes. Several groups in the world have now created spin-off companies in collaboration with hospitals to design adaptive optics systems to image the retina or to focus a laser on the retina. This is a breakthrough for early diagnosis and curing retina diseases.

Adaptive optics is a good example of a technical development that was initiated by astronomers who discovered the problem, and established the theoretical bases to solve it. But they were not able to built an operational system until the declassification of infrared detectors and deformable mirrors and all the parallel work performed for defense purposes.

\section{Detectors: the symbiotic activity}

Astronomical observations are limited by the low brightness of celestial sources. Increasing the size of the telescope was one way to improve the depth of the observations, but increasing the efficiency of the detectors is also required. Using modern detectors with efficiency close to $100 \%$ instead of photographic plates with efficiencies of a few percent provides the same gain than observing with a 5 -m diameter telescope instead of a 1-m telescope. With the exception of a few domains such as radio astronomy receivers, the technology in use to build a detector is beyond the capabilities of academic laboratories. Astronomers have always worked in collaboration with industry to develop detectors matching their needs. Such collaborations started in the beginning of the last century with the collaboration between Kodak research laboratories and astronomers. This led to the development of very high speed plates, which have been the bases for the development of all high-speed films used by photographers. Other developments to ensure sharp resolution and fine grains on large surfaces developed for Schmitt telescopes have been used later for medical X-ray radiography. This collaboration has been active until the 80 s and was stopped only by the emergence of a new generation of electronic sensors matching astronomical requirements.

Bell Labs engineers invented the charge coupled devices (CCDs) in 1969 as memory devices. Such devices looked also promising for imaging purposes and very quickly linear imaging devices were built and successfully tested, and several companies started designing imaging CCDs. The optimisation of the performance of CCDs for imaging 
was boosted by NASA's decision to have a CCD camera on board the Hubble Space Telescope. The first CCD cameras on ground-based telescopes were operational in 1980 and, in less than 10 years, CCDs have replaced almost all previously-used sensors in ground-based astronomy. The CCDs of the first generation had many problems such as poor transfer efficiency, remanence after a saturation, high dark current, etc. All these defects were more apparent in astronomical CCD cameras, working at low fluxes and in cryogenic conditions. And these astronomical cameras became the best testbed to understand the physics of the CCDs (Janesick et al. 1987). The work done in astronomy laboratories working closely with the engineers in industry to optimise the performances of these devices was essential to design new generation CCDs with better performances. Thinning the CCDs, a technique invented by astronomers, opened the possibility to make UV images and to improve the quantum efficiency of the CCDs in the blue and green. The manufacturing process improvement done to decrease the number of traps and to increase the transfer efficiency opened the possibility to decrease the pixel size. This was very useful to decrease the production costs, by increasing the production yield. The boom in digital camera using CCDs was indeed partly due to an improvement of the manufacturing process thanks to the work done in common by astronomers and industry to optimise the performances of the CCDs. Similar improvements have been obtained based on X-ray astronomy detectors spin-offs that are used in many applications, ranging from control scanners to medical imaging.

The first incentives for the development of IR detectors were defense applications. But, as it was the case with CCDs, the development of IR cameras for astronomy has been helpful for industry and defense engineers to improve the sensitivity of the detectors, to extend the wavelength domain and to move towards large detectors. Due to the cost of these detectors, defense programs funded most of the developments, and detectors used for astronomy were obtained as side products of these defense projects. But the present generation of infrared detectors are becoming affordable for many civil applications: night vision and security, thermography, medical imaging for example to look for cancerous tumors. One of the most spectacular applications of such infrared cameras is the possibility to identify persons suffering from a disease, anywhere in a crowd, by looking at their body temperature through their infrared emission. It is very likely that, in the future, the civil market will become the driver for the evolution of IR detectors, as it was the case for most of the CCD developments.

I will use one of the developments done in my home institute, the CEA (French Atomic Energy Agency) to enlighten the collaboration between industry and astronomy laboratories and how they are working together to develop new devices (Agnese et al. 2003). Until the 90s, most of mid-infrared detectors (in the 10-20 microns domain) were based on semiconductors whereby the photoelectric effect converts light into an electric current. They have a very good sensitivity, but required to be operated at cryogenic temperatures, below $20 \mathrm{~K}$. This is a strong difficulty for daily operations since cryogenic systems are costly and require trained users. But instead of semiconductors, it was also possible to use bolometers, in which the incident light is used to heat a thermometer, associated with readout electronics probing the temperature of the thermometer. The incident light heats the thermometer, and the readout electronics provides a signal proportional to the incident flux. Progress in silicon etching and microelectronics made possible the design of bidimensional arrays of bolometers. Each bolometer is a very thin membrane of silicon, only a few microns thick, supported from the main structure by insulating rods. Such detectors can be operated at room temperatures with sensitivities good enough to measure temperature differences of 0.1 degrees. Called microbolometers, they are now used in many thermal infrared cameras. TV formats, e.g. $500 \times 500$ pixels, are now available at 
a price compatible with mass market. The first microbolometers were developed in the USA, but soon after an applied research department from CEA in Grenoble, the LETI, was able to manufacture a microbolometer camera. These microbolometers are now built and commercialised by a spin-off company. At that time, in 1995, we were looking for a detector in the far-infrared domain (200 microns) to be used on the Herschel Space Observatory, an European Space Agency satellite using a $3.5 \mathrm{~m}$ diameter telescope and dedicated to far infrared and sub-millimeter observations. After discussion with LETI engineers, we decided to move to a microbolometer array, using most of the technology developed previously, but optimising the design for operations at longer wavelengths. The first difference for a far-infrared bolometer was the need to operate at very low temperatures, $300 \mathrm{mK}$ in this case. But the main technical challenge was to increase the size of the pixel from 30 microns to 750 microns, which meant etching very large suspended membranes. During the development phase, the detector manufacturing process was done in Grenoble, while in Saclay we took the lead in all the test equipments and the characterisation of the detectors. And, of course, we had continuous interaction between the 2 groups. The flight instrument has been delivered to ESA in 2007, and Herschel was successfully launched on 14 May 2009†. The first images were obtained very soon after the launch and demonstrated the capabilities of these detectors for astronomical observations. Meanwhile, the LETI engineers were thinking to use their new capability to manufacture large suspended membranes for other purposes. The idea was to design microbolometer arrays for operation in the mm wavelength domain (microwaves) instead and also at room temperature. Most materials are transparent to these wavelengths. A camera using such millimeter microbolometers can be used for many applications such as looking for landmines, industrial control, or security scanners. These millimeter wave scanners already exist but they are very expensive, and difficult to operate. A micro bolometer camera would be as easy to use as a normal TV camera. The main limitation of such cameras would actually be privacy concerns, since a person looks entirely naked when seen through a millimeter scanner. Dispatching security scanners everywhere will be limited by the acceptance of the public.

These developments are a good illustration of the interaction between applied research developing new technologies with immediate goals and astronomy, which pushes these technologies to their extreme limits. The new developments for astronomy are later injected back in the applied research field, and are at the origin of improvements in manufacturing processes or even the source for new applications.

All these examples show the complexity of the relationships between technology developments and astronomy. Astronomy has contributed to technology advances in many areas: antennas, telescopes and focusing optics spanning the entire electromagnetic spectrum, cryogenic and vacuum techniques, detectors and sensors, signal and image processing, communication technology, etc. Astronomy is technology driven, but it also drives the technology. A good summary of these relations is given in the Astronomy and Astrophysics in the new Millennium report from the US National Academy of Science : In some areas, astronomers have pioneered the technology, while in the others they have worked symbiotically with industry and the defense sector in developing and perfecting the appropriate technologies. 


\section{Global economic impact of astronomy}

Astronomical research relies on large infrastructures: very large telescopes, radio telescopes, satellites. The costs of these infrastructures are large compared to everyday life expenses. In Table 1, I compare the costs of some astronomical projects with other equipments.

Table 1. Costs of astronomical projects in comparison with non-astronomical projects

\begin{tabular}{lrl}
\hline Approximate costs (M€) of large astronomical facilities \\
\hline ALMA & 750 Ground-based mm interferometer \\
Herschel + Planck (ESA) & 100 Cost for ESA only \\
European Extremely Large Telescope (ESO) & 900 Cost for ESO only \\
\hline Indicative costs for comparison & & \\
\hline Aircraft carrier & 300 & \\
Fighter aircraft & 300 Fully operational \\
Airbus A380 & 250 & \\
Airbus A320 & 60 & \\
Highway $(100 \mathrm{~km})$ & 600 & \\
\hline
\end{tabular}

A large fraction of the construction costs of these facilities is used to place contracts for services and equipments to industry. This is a direct transfer, which is easy to quantify. A good estimate of the overall budget for European Astronomy has been obtained in 2008 during the elaboration of the ASTRONET Infrastructure Road Map $\dagger$. It is summarised in Table 2.

Table 2. Annual budget for European astronomy (M€/year)

\begin{tabular}{lr}
\hline European Southern Observatory (ESO) (member state contribution) & 150 \\
Cost of national ground-based facilities & 250 \\
European Space Agency (ESA) scientific program (member state contribution) & 400 \\
Cost of national contribution to ESA payload and national space projects & 200 \\
Cost of scientific staff and laboratories & 1000 \\
\hline Total & 2000 \\
\hline Expenses in industrial contracts & 500 \\
\hline
\end{tabular}

Dealing with the most costly infrastructures, ESO and ESA are the main providers of large industrial contracts. For both organisations, about half of the member states contributions return as expenditure in industry. Most of these expenditures are linked with high added-value activities: research and development in preparation of new projects or manufacturing challenging infrastructures like large telescopes or scientific satellites. Only about $1 / 3$ of the expenditures are the running costs to operate existing facilities: services, maintenance, transportation, etc. The situation is different for national institutes where the main expenses are associated to manpower and running costs. But even in this case, the industrial returns are far from negligible.

The economic weight of fundamental research in general, and astronomy in particular, is much larger than the direct industrial return. It is a strong stimulus for demanding products beyond existing capabilities. Nordberg (1994) has identified 14 different types of benefits resulting from industrial collaborations between industry and CERN, the 
European organisation for particle physics. As expected, improvements of manufacturing methods, research and developments or improvement of skills by tackling novel problems are in this list. More unexpected are the improvements on quality assurance or in project control, marketing benefits in terms of image, and in the increase of staff motivation through new challenges and non-conventional work. Autio et al. (1996) concluded that the main advantages for industrial companies are not the financial gain, but the benefits in the image of the company and the overall gain in skill and motivation of the employees. This is much more difficult to quantify than the direct financial gain, but turns to be more important for the benefits of industry. Here we can quote Reynald Seznec, CEO of Thales Alenia Space, in his introductory talk during the opening ceremony for the International Year of Astronomy: Astronomy projects are challenging projects able to motivate industrial teams.

Beyond industry, astronomy has a strong impact on economical activities. Two detailed studies have been performed in Hawaii and in Arizona to quantify the impact of astronomy-related activities on the public economy. Arizona was the main US location for astronomy and space science since the early beginnings of Lowell Observatory in Flagstaff in 1896. It is still a major place, with several large telescopes and many public research organisations funded mostly by NASA and NSF. In addition, many small companies dedicated to optics and instrumentation have developed over the years, mostly to respond to the needs of research institutes. Taking into account many different direct and indirect economic impacts, Pavlakovich-Kochi et al. (2007) have shown the importance of the activity created by astronomy and space science projects for the economy of the state of Arizona. They have also demonstrated that for 10 direct jobs in a public organisation devoted to astronomy and space science, 6 more jobs are generated in the Arizona economy, and that for every dollar of direct wages and salaries spent in these public organisations, 1.3 dollars are generated throughout the state's economy. Similar studies done at the level of CERN and ESA organisations (see Llewellyn Smith (1997) and references therein) have shown that for most of Big Science organisations a multiplier factor -that is, the overall gain for economy expressed as the ratio of euros generated from contracts awarded over the euros spent from public funding- is between 2 to 3 , and a multiplier between 1.2 and 1.6 when normalised by the total organisation budget. Using these multipliers and the data from Table 2 we get that the overall impact of astronomy and space science in Europe -beyond direct salaries of academic institutions and the contributions to European organisations (ESO and ESA)- has 2 main components: the expenditure through contracts to industry is of the order of $500 \mathrm{M} € /$ year, and in addition $1000 \mathrm{M} € /$ year is generated in the European economy.

The state of Hawaii is now the most prominent location for ground-based astronomy, with a concentration of large telescopes on Mauna Kea in Hawaii, and Haleakala in Maui. Astronomy has been identified by the Hawaii Science and Technology Institute as one of the main drivers for technology and innovation in the islands. For the island of Hawaii, astronomy not only brings in numerous visitors and researcher to the island, but also creates a substantial numbers of high-paying, high-skilled jobs $\dagger$. Astronomy is also particularly popular in the University of Hawaii in Hilo, and is used to develop skills in science and technology for many students. In many countries, like India, Canadaf

$\dagger$ Innovation and technology in Hawaii: An economic and workforce profile, October 2008, The Hawaii Science and Technology Institute. See www.hiscitech.org

$\ddagger$ Building on Canada's Excellence in Astronomy, presented by the Coalition for Canadian Astronomy to the House of Commons Standing Committee on Finance, 2005, www. astro.utoronto.ca/ acura/en/resources/coalitionnewsletter_en_spring_2006.pdf 
or Australia $\uparrow$, the national strategic plan for the development of astronomy emphasizes the strong benefits for industry and technology of a vigorous development of astronomy. This is also a major item in the European Road Map for Astronomy elaborated by the ASTRONET network.

\section{Conclusion}

The main goal of astronomy is pure science. But astronomy is also a Big Science, with a strong dependence on technology. Progress in astronomical observation capabilities is technology-driven. But the interactions between technology and astronomy are far from simple. The examples described in this paper are representative of this complex interaction. Beyond its main fundamental goals, most of astronomy advocates insist on the impact of astronomy to raise interest of public and students alike for science. I hope that this paper will contribute to demonstrate the importance of astronomy for industry, high-technology development and, more generally, its large economic impact.

\section{Acknowledgements}

While I have a long-standing interest in the interactions between fundamental research and technology development, my own research in this field started when I read the illuminating paper from the former CERN director, Sir Christopher Llewellyn Smith, based on a colloquium he gave at CERN on June 12, 1997 (Llellewyn Smith 1997). Many ideas developed in the present paper are already present in Llewellyn Smith's paper and the references quoted there were the starting point of my own bibliographic research.

\section{References}

Agnese, P. Cigna, C., Pornin, J. L., Accomo, R., Bonnin, C., Colombel, N., Delcourt, M., Doumayrou, E., Lepennec, J., Martignac, J., Reveret, V., Rodriguez, L., \& Vigroux, L. 2003, Proc. SPIE, 4855, 108

Autio, E., Hameri, A. P., \& Nordberg, M. 1996, J. Eng. Technol. Manag., 13, 301

Babcock, H. W. 1953, PASP, 65, 229

Janesick, J. R., Elliott, T., Collins, S., Blouke, M. M., \& Freeman, J. 1987, Optical Engineering, 26,692

Lequeux, J. 2005, L'Univers dévoilé (Les Ulis: EDP Sciences)

Llewellyn Smith, C. 1997, The use of basic science http://public.web.cern.ch/public/en/ About/BasicScience1-en.html

Nordberg, M. 1994, Contract benefits and competence-based supplier strategies: CERN as a case study (Helsinky: Institute of Industrial management, and Espoo: University of technology)

Pavlakovich-Kochi, V., Charney, A. H., \& Mwaniki-Lyman, L. 2007, Astronomy, planetary and space science research in Arizona (Tucson: Economic and Business research center, Eller College of Management, The University of Arizona)

van Woerden, H. \& Strom, R. G. 2006, Journal of Astronomical History and Heritage, 9, 3

ฯ Astronomy Australia, Prime Minister's Science, Engineering and Innovation Council, Twelfth meeting, June 2004. See http:innovation.gov.au/ScienceAndResearch 